\title{
Modern morphological engineering techniques for improving productivity of filamentous fungi in submerged cultures
}

\author{
Anna Antecka $^{1}$ (D) $\cdot$ Marcin Bizukojc $^{1} \cdot$ Stanislaw Ledakowicz $^{1}$
}

Received: 20 July 2016/Accepted: 24 September 2016/Published online: 7 October 2016

(C) The Author(s) 2016. This article is published with open access at Springerlink.com

\begin{abstract}
Morphological engineering techniques have recently gained popularity as they are used for increasing the productivity of a variety of metabolites and enzymes in fungi growing in submerged cultures. Their action is mainly associated with the changes they evoke in fungal morphology. Traditional morphological engineering approaches include manipulation of spore concentration, $\mathrm{pH}$-shifting and mechanical stress exerted by stirring and aeration. As the traditional methods proved to be insufficient, modern techniques such as changes of medium osmolality or addition of mineral microparticles to the media (microparticle-enhanced cultivation, MPEC) were proposed. Despite the fact that this area of knowledge is still being developed, there are a fair amount of scientific articles concerning the cultivations of filamentous fungi with the use of these techniques. It was described that in Ascomycetes fungi both MPEC or change of osmolality successfully led to the change of mycelial morphology, which appeared to be favorable for increased productivity of secondary metabolites and enzymes. There are also limited but very promising reports involving the successful application of MPEC with Basidiomycetes species. Despite the fact that the mineral microparticles behave differently for various microorganisms, being strain and particle specific, the low cost of its application is a great benefit. This paper reviews the application of the modern morphology engineering techniques. The authors critically assess the advantages, shortcomings, and future prospects of their application in the cultivation of fungi.
\end{abstract}

Anna Antecka

anna.antecka@p.lodz.pl

1 Department of Bioprocess Engineering, Lodz University of Technology, ul. Wolczanska 213, 90-924 Lodz, Poland
Keywords Biomass · Filamentous fungi · Medium osmolality · Microparticle-enhanced cultivation · Productivity

\section{Introduction}

Filamentous fungi are metabolically versatile microorganisms with a very wide distribution in nature (Archer et al. 2008). They secrete a variety of hydrolytic enzymes that degrade waste organic materials, and many of them are regarded as primary decomposers in nature. They are also able to produce such secondary metabolites as polyketides, alkaloids or peptides. Therefore, an increasing range of fungal species are exploited commercially as sources of enzymes and metabolites in a broad range of industrial processes from food to pharmaceutical applications (Ghaffari-Moghaddam et al. 2014; Marinelli and Marcone 2011). Moreover, the recent availability of fungal genomes has provided a major opportunity to explore and further exploit fungi as a source of enzymes and metabolites. Due to their highly efficient secretion of proteins filamentous fungi are one of the most important cell factories in the production of industrial enzymes. In an industrial context, fungal productivity depends strongly on morphology, although not always directly (Cox et al. 1998).

Filamentous microorganisms present a diverse and complicated range of gross and internal morphologies, which to date remain one of the most intriguing subjects in this field of study. Typically, they consist of hyphae, which are relatively long compared to their width, often branched, and forming extended structures called mycelia (Cox et al. 1998). There are generally two morphological forms of filamentous fungi growth: dispersed morphology and spherical hyphal aggregates, referred to as macroscopic 
pellets. This diversity of specific morphological forms of filamentous fungi, ranging from the dense spherical pellets to viscous mycelial suspensions, makes high demands on control in submerged cultivations. It is strongly dependent on culture conditions and strictly correlates with the optimal biosynthesis of the desired products. Furthermore, the existence of various morphological forms in the submerged cultures is connected with several engineering issues that have to be solved. In the macroscopic spherical pellets which can reach a diameter of several millimeters limitations in diffusive mass transfer occur, lowering nutrients and oxygen levels, especially in the centers of pellets. On the other hand, the mycelial suspension (dispersed morphology) often decreases mixing efficiency as the viscosity increases to such an extent that it makes stirring difficult. In addition, the convective oxygen transport due to strongly non-Newtonian rheological properties of the cultivation broth is limited; and it is often not clear which morphological form is favorable for the efficient production of metabolites or enzymes.

Fungal morphology can be controlled by means of genetic modifications (McIntyre et al. 2001) and other techniques that utilize the influence of cultivation conditions on fungal morphology. All the actions aimed at controlling fungal morphology are referred to as morphological engineering. According to McIntyre et al. (2001) the term is defined as "tailoring morphologies for specific bioprocesses".

In order to control fungal morphology at the process level, such traditional methods as varying spore concentration, changing $\mathrm{pH}$ level, inducing mechanical stress due to stirring and aeration, changing cultivation temperature and medium composition are implemented (Nielsen et al. 1995; Papagianni 2004; Bizukojc and Ledakowicz 2010). But in many cases they are insufficient, and thus other methods were proposed. These "new approaches" include the addition of microparticles and variation of medium osmolality (Krull et al. 2013).

Various mechanisms of pellet formation have been observed in filamentous microorganisms. One may distinguish spore agglomeration typical for Aspergilli, non-agglomerative pellet formation in Zygomycetes and Streptomycetes and mycelial agglomeration in Penicilli. In most cases the actions undertaken by morphological engineering techniques are to interfere with agglomerate (pellet) formation and produce smaller, less dense pellets or even dispersed mycelium. Such conditions often lead to better consumption of substrates and increase of fungal productivity (Kaup et al. 2008; Driouch et al. 2010a; Gonciarz and Bizukojc 2014).

There are many original papers concerning morphological engineering techniques but there are not too many reviews on this topic. As the latest review by Krull et al. was published in 2013, and this area of research develops rapidly, there is a need to review the latest findings. Therefore, the aim of this review is to present the applications of modern morphological engineering techniques in the cultivation of filamentous fungi.

\section{Microparticle-enhanced cultivation of filamentous fungi}

\section{Microorganisms: Which filamentous fungi?}

As the one of the most commonly used fungi are those of the genus Aspergillus, therefore the study concerning microparticle-enhanced cultivation (MPEC) methods were mostly concentrated on this genus. Filamentous fungi belonging to the genus Aspergillus are claimed to be one of the most intriguing and often uncontrollable organisms because of their complex morphology (Wucherpfennig et al. 2011). With regard to MPEC they were deeply studied by Driouch et al. (2010a, b, 2012). They used two recombinant strains: an uridine auxotropic, $\alpha$-glucoamylase-producing strain Aspergillus niger AB 1.13 and A. niger SKAn 1015 with $\beta$-fructofuranosidase gene (Driouch et al. 2010a). First, they studied the effect of talc and aluminum oxide microparticles on the formation of the aforementioned enzymes. Next, they made bioprocess optimization of $\beta$ fructofuranosidase production with the addition of talc microparticles in a 3-1 stirred tank bioreactor operating in the fed-batch mode (Driouch et al. 2010b). Ultimately, they used titanium silicate oxide microparticles in the cultivation of these two fungal species (Driouch et al. 2012).

Gonciarz and Bizukojc (2014) and Gonciarz et al. (2016) studied lovastatin-producing Aspergillus terreus ATCC 20542 and cultivated it in shake flasks with talc microparticles (Gonciarz and Bizukojc 2014), and in batch and continuous fed-batch stirred-tank bioreactors (Gonciarz et al. 2016). Similarly, Coban et al. (2015a, b), who studied the enhancement of phytase production by Aspergillus ficuum NRRL 3135 with addition of talc or aluminum oxide, started with shake flasks and then continued in fed-batch and continuous bioreactors. This team later changed their interests to lactic acid production by Rhizopus oryzae NRRL 395 (Coban and Demirci 2016). In the meantime, Etschmann et al. (2015) studied two fungal strains: A. niger DSM 821, being a 2-phenylethanol producer and Trichoderma atroviridae IMI 206040 a 6-pentyl$\alpha$-pyrone producer. Quite recently Aspergillus sojae AsT1, was studied by Yatmaz et al. (2016) to enhance $\beta$-mannanase production by addition of talc and aluminum oxide in the shake flask scale.

Of other fungi studied, there were three strains which do not produce asexual spores, but their morphology was also 
changed after addition of microparticles to the cultivation medium. Caldariomyces fumago DSM 1256, a chloroperoxidase producer, was the first species with which MPEC method was used (Kaup et al. 2008). Finally, two basidiomycetes strains Cerrena unicolor (Bull. Ex Fr.) Murr. 137 and Pleurotus sapidus DSM 8266 (Antecka et al. 2016) were studied to enhance laccase production by employment of MPEC.

Besides the aforementioned most profound studies, simple screening experiments were made with six other fungal species: Penicillium digitatum, Penicillium chrysogenum, Emericella nidulans, Acremonium chrysogenum, Rhizopus oryzae, Chaetomium globosum (Kaup et al. 2008).

\section{Microparticles: Which ones and how much?}

MPEC was conceptualized following a publication by Kaup et al. (2008) in which their results showed a 10-fold increase of chloroperoxidase activity in the culture medium of $C$. fumago DSM 1256 with talc microparticles added. But it did not indicate that all microparticles act in the same way. In the experiments, Kaup et al. (2008) thoroughly examined nine different concentrations (ranging from 0.05 to $25 \mathrm{~g} \mathrm{l}^{-1}$ ) of aluminum oxide and talc powder with various particle size distribution. They obtained positive results, but not in each case. The addition of microparticles at concentrations of $15 \mathrm{~g} \mathrm{l}^{-1}$ for aluminum oxide and $10 \mathrm{~g} \mathrm{l}^{-1}$ for talc powder led to the increase of biomass formation and chloroperoxidase activity, whereas lower microparticle concentrations stimulated biomass formation to a lesser degree and the higher ones did not significantly enhance biomass and product formation. Additionally, it was proven that only microparticles of a diameter smaller than $42 \mu \mathrm{m}$ could change $C$. fumago DSM 1256 morphology from pellet to dispersed. Particles of $500 \mu \mathrm{m}$ diameter did not exert any effect on growth, morphology or production of the enzyme by the fungus. Generally, microparticles of a diameter comparable to the size of spores or spore agglomerates are the most efficient.

Further investigations showed that talc microparticles have been the most often applied. The addition of these microparticles was also studied by Driouch et al. (2010a, b), Gonciarz and Bizukojc (2014), Etschmann et al. (2015) and Coban et al. (2015a). Their results confirmed that the optimal concentration of talc microparticles is about 10-20 $\mathrm{g}^{-1}$. However, it has recently been observed that talc powder is not completely inert in the medium as it releases such amounts of magnesium ions into culture media that they can be physiologically significant. The effect of biomass and productivity enhancement could be partially connected with magnesium ions (Etschmann et al. 2015). The second most popular microparticle is aluminum oxide, which proved to be completely insoluble in water and inactive (Etschmann et al. 2015). Results of other authors confirmed that the most effective aluminum oxide concentration is similar to that of talc, approximately 15-20 $\mathrm{g}^{-1}$ (Driouch et al. 2010a, b; Etschmann et al. 2015; Coban et al. 2015a; Antecka et al. 2016).

Driouch et al. (2012) also applied titanium silicate oxide $(8 \mu \mathrm{m})$ to stimulate $\alpha$-glucoamylase by $A$. niger $\mathrm{AB} 1.13$ and $\beta$-fructofuranosidase formation by A. niger SKAn 1015 and tested microparticle concentrations in the range from 5 to $50 \mathrm{~g} \mathrm{l}^{-1}$. In this case, $25 \mathrm{~g} \mathrm{l}^{-1}$ of titanate was an optimum dose. Finally, Etschmann et al. (2015) screened 16 various microparticles of different chemical composition and nominal particle diameter ranging from 5 to $250 \mu \mathrm{m}$. Their conclusions were partially unsatisfying, concluding that there is no real rule for MPEC and all the effects are strain-specific, so individual study for each case is required.

\section{Effects of MPEC: What are the targets?}

\section{Change of mycelial morphology}

In most cases the microparticles in the culture media influence both biomass concentration and fungal morphology, namely the size, shape, and the structure of the mycelia. For example, C. fumago DSM 1256 normally grows as densely packed pellets with diameters up to $4 \mathrm{~mm}$. But in the medium containing microparticles of talc or aluminum oxide the mycelium was loosely packed and attained diameters of $0.1-0.5 \mathrm{~mm}$ along with a significant amount of single hyphae (Kaup et al. 2008). Similarly, $A$. niger SKAn 1015 forms mycelial clumps of average diameter equal to $1.7 \mathrm{~mm}$, whereas with the addition of talc or aluminum oxide the size of pellets decreased to $0.1 \mathrm{~mm}$, becoming free mycelium with increasing concentrations of microparticles (Driouch et al. 2010a). Visually homogeneous cultures without agglomerates were also achieved by Etschmann et al. (2015) for A. niger DSM 821 cultivations, in which 16 various microparticles were used and all of them led to the same dispersed morphological form. In the case of $T$. atroviride IMI 206040 the same studies resulted in pellets of various sizes but no homogeneous cultures were obtained (Etschmann et al. 2015).

For A. terreus ATCC 20542 progressively smaller pellets were obtained (Fig. 1) when increasing amounts of talc microparticles were added (Gonciarz and Bizukojc 2014).

In contrast, with the addition of titanate oxide the results were different. The pellet size was almost unchanged up to $5 \mathrm{~g} \mathrm{l}^{-1}$ titanate but higher microparticle doses led to the formation of core-shell pellets (Driouch et al. 2012), which was actually a discovery of a new morphological form of A. niger SKAn 1015. The formed microparticle kernels 

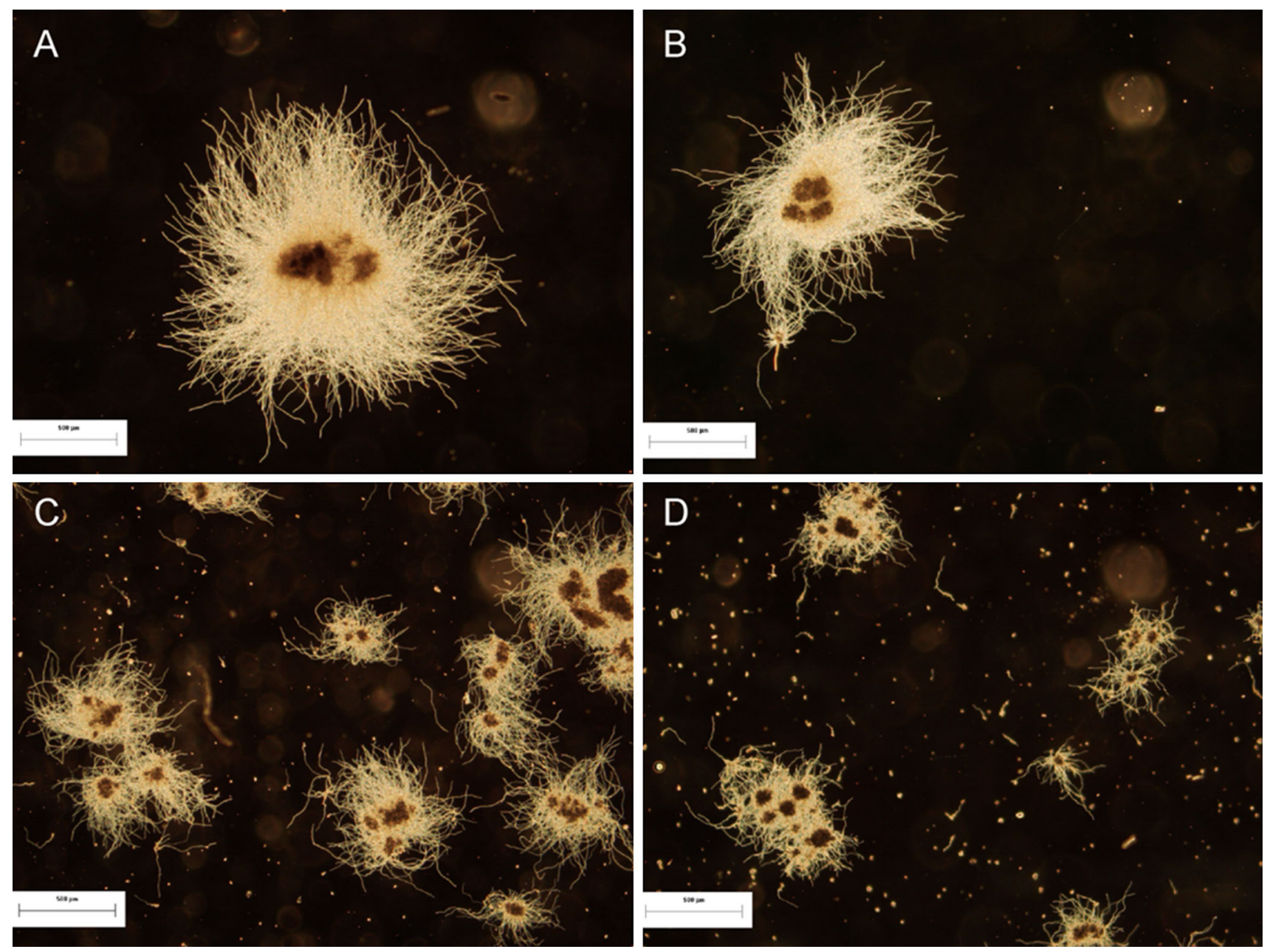

Fig. 1 Images of pellets obtained at various talc microparticles concentrations ( $24 \mathrm{~h}$ of the preculture): a control, b $1 \mathrm{~g} \mathrm{1^{-1 }}$, c $6 \mathrm{~g} \mathrm{1^{-1 }}$ and d $15 \mathrm{~g} \mathrm{l}^{-1}$; reproduced from Gonciarz and Bizukojc (2014) with permission granted from Wiley

provided solid support for the growth of biomass which during the process gradually covered the titanate cores with a thin layer of mycelium.

Coban et al. (2015a, b) reported that the addition of microparticles to the A. ficcum NRRL 3135 culture decreased average fungal pellet size and prevented bulk fungal growth. In shake flask cultivations the average fungal pellet radius decreased from $800 \mu \mathrm{m}$ for control to 500 and $200 \mu \mathrm{m}$ by increasing the particle concentration to $15 \mathrm{~g}^{-1}$ of aluminum oxide and talc, respectively. The addition of talc microparticles to the preculture of A. terreus ATCC 20542 in a stirred tank bioreactor (Gonciarz et al. 2016) also promoted the formation of smaller pellets. Finally, rare shapes of pellets called star-shaped pellets (Fig. 2) were obtained during cultivation of the basidiomycetes strain $C$. unicolor in a culture with the addition of $15 \mathrm{~g} \mathrm{l}^{-1}$ of aluminum oxide (Antecka et al. 2016). Surprisingly, another basidiomycetes strain, P. sapidus, changed its morphology in an all together different way after the addition of microparticles, and core-shell pellets, similar to the biomass of $A$. niger SKAn 1015 after the use of titanate microparticles, were achieved.

\section{Change of oxygen transfer conditions and viscosity}

One of the positive effects of MPEC is the improvement of oxygen transfer to the fungal cells due to formation of smaller pellets (Walisko et al. 2012; Driouch et al. 2010a; Gonciarz and Bizukojc 2014). Formation of mycelial clumps by filamentous fungi often causes difficulties with mixing and limitations in the diffusion of oxygen and nutrient supply to biomass (Gibbs et al. 2000), which reduce the capacity of microorganisms for growth and metabolite or enzyme production. By applying MPEC these difficulties can be significantly reduced or even eliminated as this method facilitates easier diffusion of oxygen to smaller fungal pellets. Gonciarz and Bizukojc (2014) proved that the positive effect of MPEC in lovastatin-producing A. terreus ATCC 20542 was associated with better aeration of fungal pellets, documented by the 


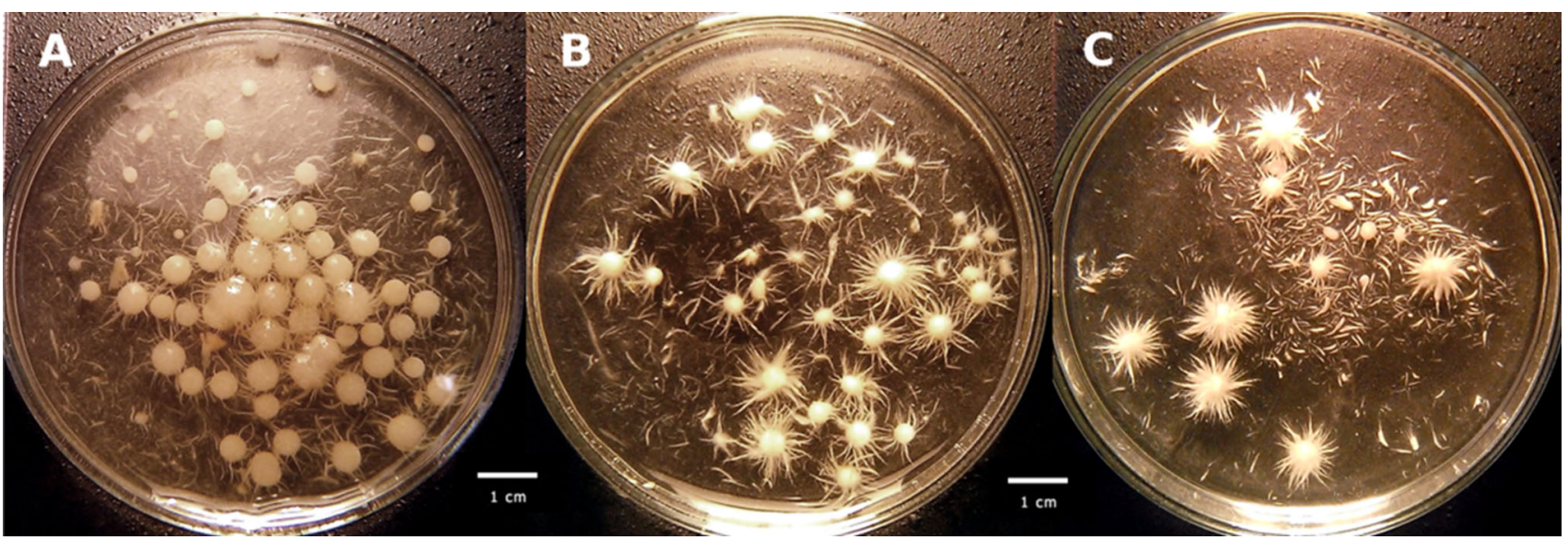

Fig. 2 Influence of aluminum oxide microparticles added to the culture medium on $C$. unicolor morphology: a control run without microparticles, b $15 \mathrm{~g}^{-1}$ and c $30 \mathrm{~g} \mathrm{l}^{-1}$; scale-bar size $1 \mathrm{~cm}$; from

intrapellet oxygen concentration profiles. In another study by means of image analysis techniques, Driouch et al. (2010a), quantitatively determined and visualized metabolically active cells of the pellets (these ones to which nutrients and oxygen reached) by means of fluorescent probes. The result of this analysis was that only a thin layer on the outer surface of the pellets remained active and capable of fluorescent protein production. Notably, this active layer was enhanced and it was evidence for how efficient MPEC could be. The interaction between microparticles and fungal mycelium created a highly active biocatalyst with the dominant fraction of cells contributing to production (Driouch et al. 2010a).

Dispersed cells are decidedly better supplied with oxygen and nutrients than large cell pellets, where the inner core can consist of essentially inactive or even dead biomass (Driouch et al. 2012). On the other hand, the formation of free mycelium increases viscosity of the broth and decreases the effective oxygen interphase transport from gas phase to liquid phase. For instance, the viscosity of free mycelium of A. niger SKAn 1015 after addition of talc was 4-fold higher than that after addition of titanate when core-shell pellets were formed (Driouch et al. 2012). That is why the optimal culture condition and morphological form of the fungi should be simultaneously optimized to ensure high product secretion while at the same time maintaining low viscosity of the culture.

As mentioned earlier, fungal morphology influences the rheology of the cultivation medium. However, it has a significant impact not only on mixing but also mass transfer within the culture medium. Therefore, better mass transfer could also be the reason why MPEC works in this way. For instance, Coban et al. (2015a) observed that the addition of the same amount of talc makes fungal pellets smaller compared to those with aluminum oxide. These
Antecka et al. (2016) distributed under the terms of the Creative Commons Attribution 4.0 International License

authors suggested that it was caused by the differences in mean particle sizes and shapes of these two different microparticles. Talc microparticles had a smaller diameter than those of aluminum oxide and provided better mass transfer in the culture medium. Additionally, one should take into account that talc is not completely inert (Etschmann et al. 2015). However, there is still no evidence whether more intensive biomass growth was caused directly by microparticles or due to microelements released from the microparticles.

\section{Change of substrate consumption rates}

Another positive effect of MPEC is the enhancement of uptake of carbon sources. It was shown by Gonciarz and Bizukojc (2014) that the presence of talc microparticles increased lactose uptake rate by lovastatin-producing $A$. terreus ATCC 20542. Substrate consumption was the slowest in the control run, and the fastest lactose uptake for talc concentration of $15 \mathrm{~g} \mathrm{l}^{-1}$ was observed within the first $24 \mathrm{~h}$ of the cultivation. On the other hand, Etschmann et al. (2015) reported that for T. atroviride IMI 206040 cultivation in the control run about $78 \%$ of the initial glucose was consumed, while in the cultures with microparticles it changed from 36 to $89 \%$ dependent on the microparticles used. So, from their results, no correlation between glucose consumption and pellet size could be established.

\section{Change of enzymes or metabolites titer}

In most cases the researchers tried to establish an optimal concentration of microparticles that could increase fungal productivity. Kaup et al. (2008) obtained about 10-fold enhancement of chloroperoxidase production by $C$. fumago DSM 1256 after addition of talc with a concentration of 


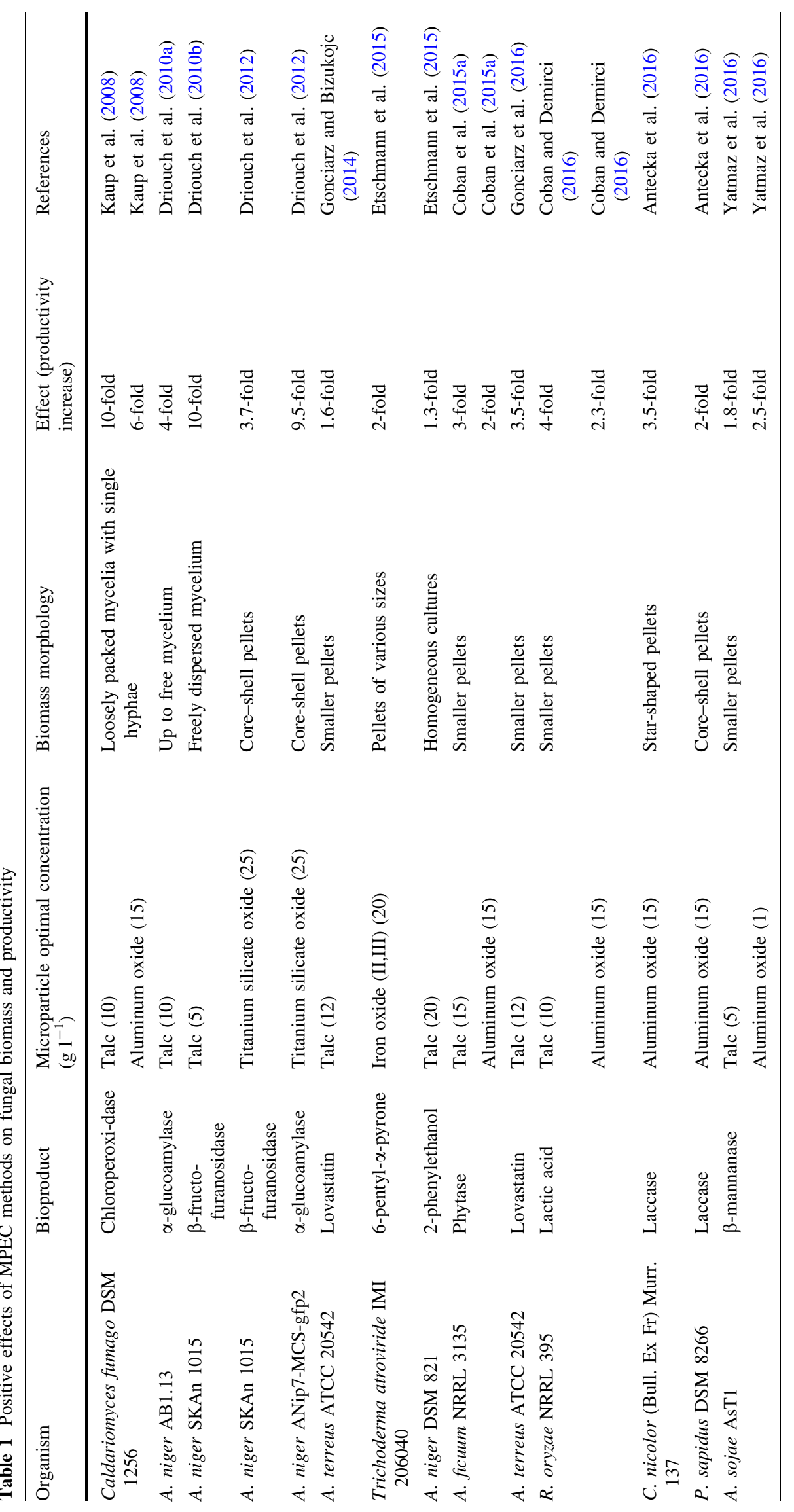


$10 \mathrm{~g} \mathrm{l}^{-1}$. Moreover, they noticed that the specific productivity of fungal biomass was also significantly enhanced. Also for organisms of the genus Aspergilli MPEC led to the increase of product formation: 4-fold higher of $\alpha$-glucoamylase and $\beta$-fructofuranosidase secretion by $A$. niger (Driouch et al. 2010a), a 3.5-fold higher lovastatin titer produced by A. terreus ATCC 20542 (Gonciarz et al. 2016) or a 3-fold increase of phytase activity by A. ficuum NRRL 3135 (Coban et al. 2015a). The latest study also proved the positive effect of microparticles for basidiomycetes strains (Antecka et al. 2016). Despite the fact that fungal morphology for $C$. unicolor and $P$. sapidus were completely different, in both cases laccase activity was increased, 3.5 and 2-fold, respectively. Table 1 brings together results of MPEC showing positive effects when applied to filamentous fungi cultivation.

When comparing the increase of product formation, it is seen that some fungi responded better to the particle addition than others. Etschmann et al. (2015) who studied above 15 various microparticles claimed that for $T$. atroviride IMI 206040 it was not possible to establish the correlation between pellet size and 6-pentyl- $\alpha$-pyrone concentration. But, in the same study, they were able to choose the group of particles which enhanced 6-pentyl- $\alpha$ pyrone formation. The highest concentration of 6-pentyl- $\alpha$ pyrone was achieved after addition of iron oxide (II, III) or aluminum titanate, which caused about 2-fold increase of 6 -pentyl- $\alpha$-pyrone titer compared to unsupplemented control. Interestingly, for 2-phenylethanol production by $A$. niger DSM 821 the best result was obtained with the addition of $40 \mu \mathrm{m}$ talc microparticles.

To sum up, it must be clearly stated that it is difficult to establish any general rules between filamentous fungi, microparticles used and the resulting fungal productivities. Each fungal species, microparticles and product should be analyzed individually in each given culture condition (Etschmann et al. 2015).

\section{How do microparticles act?}

Presently, it is still unclear why the metabolites or enzymes displayed increased productivity in MPEC than in control cultivations. In the majority of works cited in this review not much was written about the mechanism of the action of microparticles. The question remains as to how the microparticles change the morphology of fungi and, as a consequence, increase productivity of strains. Only Driouch et al. (2010a) presented a short description of the interactions between microparticles and fungal cells following microscopic observations. They monitored the morphological development of $A$. niger at various time points during the initial stages of cultivation. In the control culture large agglomerates typically observed in submerged fungal cultures were maintained and then transformed into large solid pellets. When talc microparticles were added, the initially present large spore agglomerates disappeared, and during germination only individual spores were observed, resulting in a loose mycelium. However, when the microparticles were added at later stages of cultivation the morphology of $A$. niger was not affected. This indicates that the microparticles disturbed the initial phase of spore aggregation and was the reason for the change in morphology. Therefore, finding the moment of cultivation time to add the microparticles was crucial. It was proven that in order to induce the desired morphological changes of the growing mycelium microparticles must be added together with spores when the agglomerates are just beginning to form. According to the present knowledge, one can conclude that the action of microparticles relies the surface interaction between spores and microparticles and it is rather a kind of spatial and mechanical effect (assuming fully inert microparticles). Nevertheless, further physiological effects were induced as the microparticles promoted the formation of smaller pellets and facilitated access of oxygen to the mycelium. As a result, more efficient consumption of substrates and increase of fungal productivity were often observed (Driouch et al. 2010a, b; Gonciarz and Bizukojc 2014; Coban et al. 2015a).

\section{Changes of medium osmolality}

Osmolality of the solution is defined as the number of moles of osmotically active substances per $1 \mathrm{~kg}$ of solvent. In microbiology this feature of the solution or cultivation medium is often referred to as water activity. Osmolality of the media strongly influences microbial growth. If it is too high, growth may completely cease. Typical osmolalities of cultivation media are between 0.28 and 0.32 osmol kg-1. Previously, it was observed that osmotic stress may induce the excretion of enzymes (Bobowicz-Lassociska and Grajek 1995; Fiedurek 1998). Common substances that can be used to cause osmotic stress are sodium and potassium chlorides, which are physiologically inert salts. Osmotic stress has rarely been proposed to control fungal morphology. In fact, Wucherpfennig et al. (2011) were the only team to do this, they proved that changing of osmolality can be the tool of morphological engineering. However, because osmolality is not as metabolism neutral as MPEC, in their study they had to distinguish the metabolic effect of sodium chloride from the morphological one. Wucherpfennig et al. (2011) studied two strains, previously studied in MPEC, A. niger SKAn1015 ( $\beta$-fructofuranosidase producer) and AB1.13 ( $\alpha$-glucoamylase producer). They used a 3-1 working volume stirred-tank 
bioreactor. The basic medium for A. niger SKAn1015 had an osmolality of $0.4 \mathrm{osmol} \mathrm{kg} \mathrm{k}^{-1}$ and the study was made in the range of $0.4-4.9 \mathrm{osmol} \mathrm{kg}^{-1}$. The maximum of fructofuranosidase activity $\left(220 \mathrm{U} \mathrm{ml}^{-1}\right)$ was found between 2.6 and $3.2 \mathrm{osmol} \mathrm{kg}^{-1}$. In the case of glucoamylase production by $A$. niger $\mathrm{AB} 1.13$ no optimum osmolality was found. Enzymatic activity achieved $22 \mathrm{U} \mathrm{ml}^{-1}$ with increasing osmolality from $0.2 \mathrm{os}-$ mol kg$~^{-1}$ (basic medium) to 2.4 osmol kg-1.

Osmolality decreased the size of fungal agglomerates. For A niger SKAn1015 mean projected area of pellets decreased from $1.5 \times 10^{6} \mu \mathrm{m}^{2}$ for $0.4 \mathrm{osmol} \mathrm{kg}^{-1}$ to

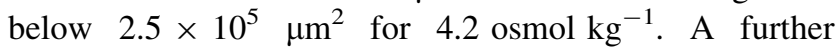
increase of sodium chloride led to the formation of dispersed mycelium. Furthermore, osmolality changed the size of pellets, which were not ideally spherical. In order to prove that changes in pellet morphology, not only osmotic stress, resulted in better production of enzymes (18-fold increase of $\beta$-fructofuranosidase activity compared to control), they proposed the original dimensionless morphology number:

Morphologynumber $=\frac{2 \cdot \sqrt{A} \cdot S}{\sqrt{\pi} \cdot D \cdot E}$

where A-projected area of the pellet $\left(\mu \mathrm{m}^{2}\right), \mathrm{S}$ - solidity of the pellet, $\mathrm{D}$-maximum diameter of the pellet $(\mu \mathrm{m}), \mathrm{E}-$ aspect ratio (length to width ratio).

Morphology number for ideally spherical pellets is equal to 1 , while for the dispersed mycelium is equal to 0 . The values in-between correlate to the variety of shapes and sizes of the pellets. Owing to this number, they could clearly show that the enzymatic specific activity (enzyme productivity) decreased exponentially with the morphology number. Dispersed mycelium proved to be more efficient. Exhibiting an inverse relationship, as pellets grew denser and more spherical (higher morphology number) less enzymes were produced.

Wucherpfennig et al. (2011) found that the increase of osmolality acted on fungal growth and subsequently fungal morphology in two ways. Firstly, it caused delayed initiation of growth, increasing the duration of lag-phase. Secondly, the duration of spore germination phase was also longer. These factors contributed to the formation of smaller pellets or dispersed mycelium. This strong effect of osmolality was also confirmed by the specific growth rate of biomass, which actually decreased by one magnitude from 0.16 to $0.02 \mathrm{~h}^{-1}$ when osmolality was changed from 0.4 to 4.9 osmol kg ${ }^{-1}$. Despite the slower evolution of the culture, Wucherpfennig et al. (2011) claimed that osmolality is a cheap and reliable approach to increase the productivity of industrial processes. Because of the predictable behavior of fungal morphology under osmotic stress, better control of the culture is possible.

\section{Summary and future studies}

MPEC and changing osmolality are modern methods, which open new possibilities for tailor-made morphology design of filamentous fungi. According to the data in available literature, it seems to be especially useful for the fungi which have an agglomerative character of pellet formation from asexual spores. However, a full understanding of the mechanism of action of microparticles requires further investigation. It is known that the method works by decreasing the diameter of macropellets, ultimately changing the morphology into dispersed hyphae; however, there is presently a lack of any quantitative data describing this phenomenon. MPEC is to a small extent dependent upon the chemical particle composition. In the literature there are some examples of correlations between morphology and fungal productivity, additionally, the positive effects of MPEC seem to be strain and particle size dependent and require individual investigations in each case. Therefore, future studies in this field must be conducted, especially the study of species other than those belonging to the genus Aspergillus, whose growth with regard to morphology (mainly the mechanism of pellet formation) is different.

The successful use of osmolality changes for controlling fungal morphology still requires further research, as only one paper (Wucherpfennig et al. 2011) was devoted to it. Nevertheless, its authors introduced a valuable tool (morphology number) to study the control of fungal cultivations with the application of morphological engineering techniques.

Lastly, from an industrial point of view, it is important that the concept of morphological engineering was successfully transferred from flasks into bioreactor (Driouch et al. 2010a, b, 2012; Coban et al. 2015b; Gonciarz et al. 2016). The relatively low costs of the method (inexpensive microparticles added in low amounts and sodium chloride) is of great relevance for future large scale applications.

Acknowledgments The work was supported by the National Science Center (Republic of Poland) within the Project Number: UMO-2013/ 11/B/ST8/00337. We would like to thank Joanna Nodzykowski for editing the English text.

Open Access This article is distributed under the terms of the Creative Commons Attribution 4.0 International License (http://crea tivecommons.org/licenses/by/4.0/), which permits unrestricted use, distribution, and reproduction in any medium, provided you give appropriate credit to the original author(s) and the source, provide a link to the Creative Commons license, and indicate if changes were made. 


\section{References}

Antecka A, Blatkiewicz M, Bizukojc M, Ledakowicz S (2016) Morphology engineering of basidiomycetes for improved laccase biosynthesis. Biotechnol Lett 38:667-672

Archer DB, Connerton IF, MacKenzie DA (2008) Filamentous fungi for production of food additives and processing aids. Adv Biochem Eng Biotechnol 111:99-147

Bizukojc M, Ledakowicz S (2010) The morphological and physiological evolution of Aspergillus terreus mycelium in the submerged culture and its relation to the formation of secondary metabolites. World J Microbiol Biotechnol 26:41-54

Bobowicz-Lassociska T, Grajek W (1995) Changes in protein secretion of Aspergillus niger caused by the reduction of the water activity by potassium chloride. Acta Biotechnol 15(3):277-287

Coban HB, Demirci A (2016) Enhancement and modeling of microparticle-added Rhizopus oryzae lactic acid production. Bioprocess Biosyst Eng 39:323-330

Coban HB, Demirci A, Turhan I (2015a) Microparticle-enhanced Aspergillus ficuum phytase production and evaluation of fungal morphology in submerged fermentation. Bioprocess Biosyst Eng 38:1075-1080

Coban HB, Demirci A, Turhan I (2015b) Enhanced Aspergillus ficuum phytase production in fed-batch and continuous fermentations in the presence of talcum microparticles. Bioprocess Biosyst Eng 38:1431-1436

Cox PW, Paul GC, Thomas CR (1998) Image analysis of the morphology of filamentous micro-organisms. Microbiology 144:817-827

Driouch H, Sommer B, Wittmann C (2010a) Morphology engineering of Aspergillus niger for improved enzyme production. Biotechnol Bioeng 105:1058-1068

Driouch H, Roth A, Dersch P, Wittmann C (2010b) Optimized bioprocess for production of fructofuranosidase by recombinant Aspergillus niger. Appl Microbiol Biotechnol 87:2011-2024

Driouch H, Hänsch R, Wucherpfennig T, Krull R, Wittmann C (2012) Improved enzyme production by bio-pellets of Aspergillus niger: targeted morphology engineering using titanate microparticles. Biotechnol Bioeng 109:462-471

Etschmann MMW, Huth I, Walisko R, Schuster J, Krull R, Holmann D, Wittmann C, Schrader J (2015) Improving 2-phenylethanol and 6-pentyl-a-pyrone production with fungi by microparticleenhanced cultivation. Yeast 32:145-157

Fiedurek J (1998) Effect of osmotic stress on glucose oxidase production and secretion by Aspergillus niger. J Basic Microbiol 38(2):107-112
Ghaffari-Moghaddam M, Eslahi H, Omay D, Zakipour-Rahimabadi E (2014) Industrial applications of enzymes. Rev J Chem 4:341-361

Gibbs PA, Seviour RJ, Schmid F (2000) Growth of filamentous fungi in submerged culture: problems and possible solutions. Crit Rev Biotechnol 20(1):17-45

Gonciarz J, Bizukojc M (2014) Adding talc microparticles to Aspergillus terreus ATCC 20542 preculture decreases fungal pellet size and improves lovastatin production. Eng Life Sci 14:190-200

Gonciarz J, Kowalska A, Bizukojc M (2016) Application of microparticle-enhanced cultivation to increase the access of oxygen to Aspergillus terreus ATCC 20542 mycelium and intensify lovastatin biosynthesis in batch and continuous fedbatch stirred tank bioreactors. Biochem Eng J 109:178-188

Kaup B, Ehrich K, Pescheck M, Schrader J (2008) Microparticleenhanced cultivation of filamentous microorganisms: increased chloroperoxidase formation by Caldariomyces fumago as an example. Biotechnol Bioeng 99:491-498

Krull R, Wucherpfennig T, Eslahpazir Esfandabadi M, Walisko R, Melzer G, Hempel D, Kampen I, Kwade A, Wittmann C (2013) Characterization and control of fungal morphology for improved production performance in biotechnology. J Biotechnol 163:112-123

Marinelli F, Marcone GL (2011) Microbial secondary metabolites. Compr Biotechnol Ind Biotechnol Commod Prod 3:285-297

McIntyre M, Müller C, Dynesen J, Nielsen J (2001) Metabolic engineering of the morphology of Aspergillus. Adv Biochem Eng/Biotechnol 73:104-128

Nielsen J, Johansen CL, Jacobsen M, Krabben P, Villadsen J (1995) Pellet formation and fragmentation in submerged cultures of Penicillium chrysogenum and its relation to penicillin production. J Biotechnol Prog 11(1):93-98

Papagianni M (2004) Fungal morphology and metabolite production in submerged mycelial processes. Biotechnol Adv 22:189-259

Walisko R, Krull R, Schrader J, Wittmann Ch (2012) Microparticle based morphology engineering of filamentous microorganisms for industrial bio-production. Biotechnol Lett 34:1975-1982

Wucherpfennig T, Hestler T, Krull R (2011) Morphology engineering-Osmolality and its effect on Aspergillus niger morphology and productivity. Microb Cell Fact 10:58

Yatmaz E, Karahalil E, Germec M, Ilgin M, Turhan I (2016) Controlling filamentous fungi morphology with microparticles to enhanced $\beta$-mannanase production. Bioprocess Biosyst Eng. doi:10.1007/s00449-016-1615-8 